\title{
Comparison of Germ Patterns and Antimicrobial Susceptibility in Chronic Suppurative Otitis Media with Cholesteatoma and without Cholesteatoma in Dr. \\ Mohammad Hoesin Hospital.
}

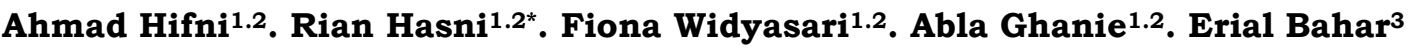 \\ ${ }^{1}$ Department of Otolaryngology. Head and Neck Surgery. Faculty of Medicine. Universitas Sriwijaya. Palembang. Indonesia \\ ${ }^{2}$ Department of Otolaryngology. Head and Neck Surgery. Faculty of Medicine. Universitas Sriwijaya. Palembang. Indonesia \\ ${ }^{3}$ Department of Anatomy. Faculty of Medicine. Universitas Sriwijaya. Indonesia
}

\section{A R T I C L E I N F O \\ Keywords: \\ CSOM \\ Germ pattern \\ Antibiotic susceptibility \\ Bacterial culture \\ *Corresponding author: \\ Rian Hasni \\ E-mail address: \\ rianhasni86.rh@gmail.com}

All authors have reviewed and approved the final version of the manuscript.

https://doi.org/10.32539/bsm.v5i7.332

\begin{abstract}
A B S T R A C T
Background: Chronic suppurative otitis media (CSOM) is a chronic inflammation of the middle ear and mastoid cavity. It is necessary to identify bacterial patterns and antibiotics susceptibility in CSOM. Appropriate administration of antibiotics is essential to treat infection and prevent widespread resistance. Objective: The aim of this study is to compare the pattern of germs and antibiotic susceptibility between CSOM with cholesteatoma and without cholesteatoma. Methods: Observational study with cross-sectional design. Data collection was carried out using medical record data on 102 subjects who underwent middle ear swab and bacterial culture from January to December 2019. Results: From 102 subjects and 122 bacterial culture results. it was found that Pseudomonas aeruginosa (36.1\%) was one of the most common organisms to cause CSOM. followed by Staphylococcus haemolyticus. Staphylococcus epidermidis and Staphylococcus aureus. There was a significant difference between the germ pattern and the type of CSOM $(p=0.002)$. Amikacin. meropenem. gentamicin. cefepime. ciprofloxacin and ceftazidime were found to be the most effective antibiotics for Pseudomonas aeruginosa. The rate of resistance to tigecycline and cefazoline were highest. Age. sex. nutritional status and comorbidities were not factors that significantly influenced the incidence of cholesteatoma in CSOM. Conclusion: There is a significant difference between the germ pattern in both CSOM groups. Patients that show Gram negative have a significant effect on the incidence of CSOM with cholesteatoma.
\end{abstract}

\section{Introduction}

Chronic suppurative otitis media (CSOM) is an important public health problem. especially in developing countries. CSOM is a chronic infection involving one or more microorganisms from the middle ear and mastoid. characterized by the discharge of ear fluid (otorrhoea) through the perforation of the tympanic membrane. CSOM is divided into two. namely chronic otitis media without cholesteatoma and with cholesteatoma. CSOM without cholesteatoma can still be treated with antibiotics and other medicaments. while CSOM with cholesteatoma needs operative management. 1.2
A survey from WHO shows that Southeast Asia has the highest number of cases of antibiotic resistance in the world. especially infections caused by Methicillin resistant Staphylococcus aureus (MRSA). resulting in decreased antibiotic function. Infectious diseases are also still a major health problem in Indonesia. In addition. it is found that $30 \%$ to $80 \%$ of antibiotic use is not based on indications. Early identification and proper management is very important especially in reducing complications associated with this disease. So far. the administration of antibiotics for CSOM is only based on the latest reports on which bacteria are most often found. whereas currently the development of 
antibiotic resistance and changes in bacterial susceptibility patterns is increasingly widespread. ${ }^{3-5}$

Treatment of people with CSOM appropriately and adequately needs to know the pattern of germs and their sensitivity to antibiotics. although the pattern of germs and their sensitivity can change from time to time and from one place to another. Through this research. it is expected to know the pattern of germs and their sensitivity to antibiotics in CSOM cases in Dr. Mohammad Hoesin (RSMH) so that the administration of antibiotics can be adjusted to the existing germ patterns until waiting for the results of culture and antibiotic sensitivity tests. in the end the infection in CSOM patients can be handled appropriately. This study aims to compare the pattern of germs and antibiotic sensitivity between CSOM with cholesteatoma and without cholesteatoma.

\section{Method}

This study is an observational study with a crosssectional design. The research was conducted at the RSMH Medical Record Installation. The research sample was the medical record data of OMSK patients who had ear swabs at the ENTKL Otology Clinic and culture and antibiotic sensitivity tests at the RSMH Microbiology Department during the period January to December 2019 and met the research criteria for analysis. Sampling in this study was carried out by total sampling.

The inclusion criteria in this study were complete medical record data from CSOM patients who had middle ear swabs and culture and antibiotic sensitivity tests conducted at the RSMH Microbiology Department. the middle ear swab culture results showed that there were germs / bacteria growing. culture results showed germs is registered in the Clinical and Laboratory Standards Institute (CLSI) guidelines at the RSMH Department of Microbiology so that antibiotic sensitivity tests can be carried out. The exclusion criteria were medical records of CSOM patients who had middle ear swabs after undergoing mastoid surgery in the last 6 weeks.

The variables in this study include independent variables. namely CSOM with cholesteatoma and
CSOM without cholesteatoma. the dependent variable is the pattern of germs and antibiotic sensitivity and confounding variables. namely age. sex. nutritional status and comorbidities. Processing and data results are assisted by SPSS software for windows version 24.0. Statistical analysis to test the comparison of germ patterns and antibiotic sensitivity in CSOM patients was performed using the Chi-Square test or Fisher's exact test.

This study has been considered ethical and approved by the Health Research Ethics Committee of Dr. Mohammad Hoesin Palembang. Ethical license is implemented in accordance with applicable procedures and rules.

\section{Results}

The number of study subjects was 102 CSOM patients consisting of $58 \mathrm{CSOM}$ patients with cholesteatoma and 44 CSOM patients without cholesteatoma. Characteristics based on age obtained the age range of the subjects between 6 years and 70 years. The majority of subjects were in the age group 19-60 years $(71.6 \%)$.

The subjects in this study were mostly male. Based on the nutritional status. it was found that the most subjects with good nutritional status were 66 people (64.7\%). 21 people (20.6\%) over nutritional status and 15 people (14.7\%) less nutritional status. Based on the presence or absence of comorbids. out of a total of 102 subjects. 23 people $(22.5 \%)$ were comorbid and 79 (77.5\%) had no comorbids.

The germ patterns were divided into Gram positive and Gram negative categories. In this study. 27 types of bacteria were identified. consisting of 11 species of Gram positive bacteria and 16 species of Gram negative bacteria. Of the 102 study subjects. a total of 122 bacteria grew. Gram negative bacteria were found more frequently. namely 70 (57.4\%). while Gram positive bacteria were 52 (42.6\%). Based on the type of CSOM. $70.3 \%$ more Gram negative bacteria were found in the CSOM group with cholesteatoma. Pseudomonas aeruginosa was the most common microorganism. This bacterium was identified in 40 patients. isolated from 44 specimens and accounted for $36.1 \%$ of all cultured 
bacteria. The other most common bacteria found are Staphylococcus haemolyticus. Staphylococcus epidermidis and Staphylococcus aureus. The frequency and distribution of the other 23 bacteria were only found in small numbers (less than 10).

The distribution of antibiotic sensitivity in Pseudomonas aeruginosa based on their sensitivity to antibiotics can be seen in Table 3 . Sensitivity tests were carried out for 10 types of antibiotics. Of all the antibiotics tested, it was found that Pseudomonas aeruginosa had the highest percentage of sensitivity to the antibiotics amikacin (93.2\%), meropenem (84.15), gentamicin $(79.5 \%)$, cefepime $(77.3 \%)$, ciprofloxacin and ceftazidime (72.7). Meanwhile, the level of antibiotic resistance was higher in tigecycline and cefazoline.

A sensitivity test was carried out against 15 types of antibiotics on Staphylococcus haemolyticus. Of all the antibiotics tested, it was found that Staphylococcus haemolyticus was very sensitive to vancomycin, nitrofurantoin and linezolide antibiotics, each of 93.3\%. These bacteria are also sensitive to gentamicin, erythromycin, tigecycline, trimethoprim / sulfamethoxazole and rifampicin. Meanwhile, there was a very high level of resistance to the antibiotics ciprofloxacin, levofloxacin, moxifloxacin, benzylpenicillin, oxacillin and tetracycline. The distribution of antibiotic sensitivity in Staphylococcus haemolyticus can be seen in Table 4.

The distribution of antibiotic sensitivity in Staphylococcus epidermidis can be seen in Table 5. A sensitivity test was performed for 15 types of antibiotics. Of all the antibiotics tested, the highest sensitivity percentage was $100 \%$ for vancomicin, nitrofurantoin, rifampicin and linezolide antibiotics. The antibiotics gentamicin and tigecycline also had a fairly high sensitivity with a percentage of $92.9 \%$. The highest level of resistance to benzylpenicillin antibiotics (100\%), whereas ciprofloxacin, levofloxacin, erythromycin, clindamycin also showed resistance to Staphylococcus epidermidis.

A sensitivity test was carried out against 15 types of antibiotics on Staphylococcus aureus with a total of 11 samples. Staphylococcus aureus in this study has high sensitivity to 10 antibiotics including ciprofloxacin, levofloxacin, moxifloxacin, gentamicin, tigecycline, trimetropine / sulfametoxsazole, rifampicin, nitrofurantoin, oxacillin and linezolide with a percentage of $100 \%$. Erythromicin, Clindamicin, Vancomicin are also considered quite sensitive with a rate of $90.9 \%$. Resistance appears to be the antibiotic benzylpenicillin. The distribution of antibiotic sensitivity in Stapylococcus aureus can be seen in Table 6.

The data from the analysis showed a $\mathrm{p}$ value of 0.002. It can be concluded that there was a significant difference between the germ patterns in the 2 CSOM groups where Gram negative bacteria were more common in the CSOM group with cholesteatoma.

Analysis of various types of antibiotics against the types of CSOM in the top 4 germs found, namely Pseudomonas aeruginosa, Staphococcus haemolyticus, Staphococcus epidermidis and Staphococcus aureus were carried out using the Fischer exact test and Chi-square Pearson test. From the results of the analysis, all showed that there was no significant effect of cholesteatoma on the level of antibiotic resistance with a $\mathrm{p}$ value $>0.05$. It can be concluded that there is no significant difference between the types of CSOM on the level of resistance to the antibiotics tested.

In the table showing the sensitivity of 10 antibiotics to Pseudomonas aeruginosa, it can be seen that the number of all antibiotics that are resistant is higher in the CSOM group with cholesteatoma than in the CSOM group without cholesteatoma. Although the results of the analysis showed that none of the antibiotics were significantly different between the two CSOM groups, both the group with cholesteatoma and the group without cholesteatoma. In the multivariate analysis, the dependent variable was cholesteatoma and the independent variables were age, sex, nutritional status, comorbid and germ groups based on the Gram positive and Gram negative division, it was found that only the germ group variable had a significant effect on the presence of cholesteatoma. OMSK patients who experienced Gram-negative bacterial infection had a significant effect on experiencing CSOM with 


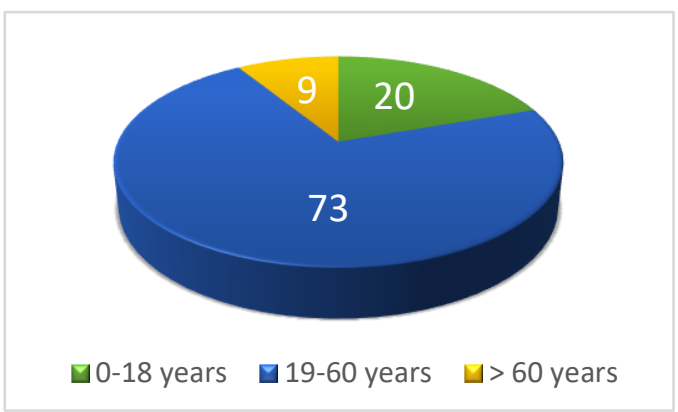

Figure 1. Distribution of research subjects by age $(\mathrm{N}=102)$

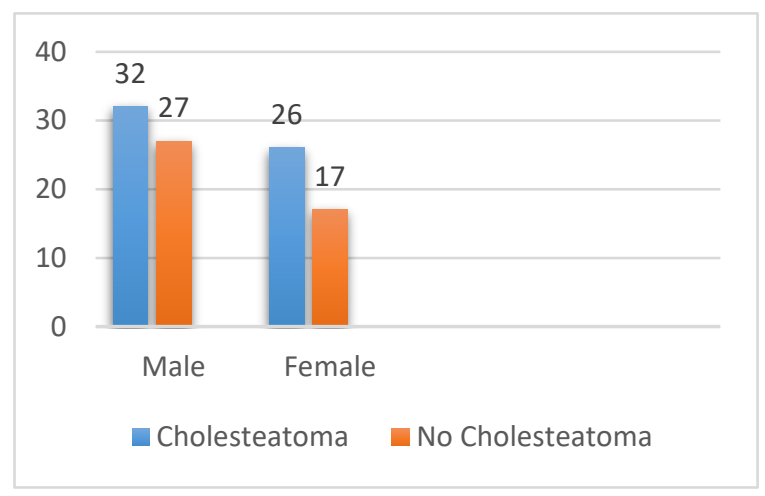

Figure 2. Distribution of research subjects based on gender $(\mathrm{N}=102)$

Table 1. Characteristics of research subjects $(\mathrm{N}=102)$

\begin{tabular}{lcc}
\hline Variable & Frequency & Percentage \\
\hline Gender & 59 & 57.8 \\
Male & 43 & 42.2 \\
Female & & \\
Nutritional status & 15 & 14.7 \\
Malnutrition & 66 & 64.7 \\
Good nutrition & 21 & 20.6 \\
More nutrition & & \\
Comorbid & 23 & 22.5 \\
Yes & 79 & 77.5 \\
No & &
\end{tabular}


Table 2. Distribution of germ patterns in CSOM $(\mathrm{N}=122)$

\begin{tabular}{lccc}
\hline Germ pattern & $\begin{array}{c}\text { CSOM with } \\
\text { Cholesteatoma N }(\%)\end{array}$ & $\begin{array}{c}\text { CSOM without } \\
\text { Cholesteatoma N }(\%)\end{array}$ & Total N(\%) \\
\hline Gram positive bacteria & & & \\
Staphylococcus haemolyticus & $2(10.5)$ & $13(39.4)$ & $15(28.8)$ \\
Staphylococcus epidermidis & $7(36.8)$ & $7(21.2)$ & $14(26.9)$ \\
Staphylococcus aureus & $4(21.1)$ & $7(21.2)$ & $11(21.2)$ \\
Staphylococcus hominis & $1(5.3)$ & $2(6.1)$ & $3(5.8)$ \\
Staphylococcus lugdunensis & $1(5.3)$ & $1(3.0)$ & $2(3.8)$ \\
Enterococcus faecalis & $2(10.5)$ & $0(0.0)$ & $2(3.8)$ \\
Staphylococcus capitis & $0(0.0)$ & $1(3.0)$ & $1(1.9)$ \\
Staphylococcus lentus & $1(5.3)$ & $0(0.0)$ & $1(1.9)$ \\
Staphylococcus. warneri & $0(0.0)$ & $1(3.0)$ & $1(1.9)$ \\
Streptococcus pyogenes & $1(5.3)$ & $0(0.0)$ & $1(1.9)$ \\
Bacillus species & $0(0.0)$ & $1(3.0)$ & $1(1.9)$ \\
\hline Total & $19(100.0)$ & $33(100.0)$ & $52(100.0)$ \\
\hline Gram negative bacteria & & & \\
Pseudomonas aeruginosa & $24(53.3)$ & $20(80.0)$ & $44(62.9)$ \\
Acinetobacter baumannii & $5(11.1)$ & $0(0.0)$ & $5(7.1)$ \\
Klebsiella pneumoniae & $4(8.9)$ & $0(0.0)$ & $4(5.7)$ \\
Sphingomonas paucimobilis & $1(2.2)$ & $2(8.0)$ & $3(4.3)$ \\
Pseudomonas Stutzeri & $2(4.4)$ & $0(0.0)$ & $2(2.9)$ \\
Proteus mirabilis & $2(4.4)$ & $0(0.0)$ & $2(2.9)$ \\
Pseudomonas fluorescens & $1(2.2)$ & $0(0.0)$ & $1(1.4)$ \\
Achromobacter denitrificans & $0(0.0)$ & $1(4.0)$ & $1(1.4)$ \\
Achromobacter xylosoxidans & $1(2.2)$ & $0(0.0)$ & $1(1.4)$ \\
Burkholderia cepacia & $0(0.0)$ & $1(4.0)$ & $1(1.4)$ \\
Citrobacter koseri & $0(0.0)$ & $1(4.0)$ & $1(1.4)$ \\
Eschericia coli & $1(2.2)$ & $0(0.0)$ & $1(1.4)$ \\
Morganella morgagnii & $1(2.2)$ & $0(0.0)$ & $1(1.4)$ \\
Providencia stuartii & $1(2.2)$ & $0(0.0)$ & $1(1.4)$ \\
Seratia odorifera & $1(2.2)$ & $0(0.0)$ & $1(1.4)$ \\
Stenotrophomonas maltophilia & $1(2.2)$ & $0(0.0)$ & $1(1.4)$ \\
\hline Total & $45(100.0)$ & $25(100.0)$ & $70(100.0)$ \\
\hline & & & \\
& & &
\end{tabular}

Table 3. Distribution of antibiotic sensitivity to pseudomonas aeruginosa $(n=44)$

\begin{tabular}{llccc}
\hline Antibiotic group & Antibiotics & Sensitive & Resistant & $\begin{array}{c}\text { Sensitive antibiotics } \\
\text { (\%) }\end{array}$ \\
& & & & 93.2 \\
Aminoglycosides & Amikacin & 41 & 3 & 79.5 \\
& Gentamicin & 35 & 9 & 84.1 \\
Carbapenem & Meropenem & 37 & 7 & 72.7 \\
Fluoroquinolones & Ciprofloxacin & 32 & 12 & 61.4 \\
Penicillin & Piperaciline / & 27 & 17 & 54.5 \\
Monobactam & Tazobactam & & & 72.7 \\
Cephalosporins & Aztreonam & 24 & 20 & 77.3 \\
& Ceftazidime & 32 & 12 & 2.3 \\
& Cefepime & 34 & 10 & 0.0 \\
\hline \multirow{2}{*}{ Tetracyclines } & Cefazoline & 1 & 43 & \\
\hline
\end{tabular}


Table 4. Distribution of antibiotic sensitivity to staphylococcus haemolyticus $(\mathrm{n}=15$ )

\begin{tabular}{llccc}
\hline Antibiotic group & Antibiotics & Sensitive & Resistant & $\begin{array}{c}\text { Sensitive antibiotics } \\
\text { (\%) }\end{array}$ \\
& & 14 & 1 & 93.3 \\
Glycopeptides & Vancomicin & 14 & 1 & 93.3 \\
Furadantin & Nitrofurantoin & 14 & 1 & 93.3 \\
Oxazolidinone & Linezolide & 12 & 3 & 80.0 \\
Aminoglycosides & Gentamicin & 12 & 3 & 80.0 \\
Trimetorpim & Trimethoprim / Sulfamethoxazole & 12 & 3 & 80.0 \\
Antimiko Bacterial & Rifampicin & 11 & 4 & 73.3 \\
Macrolides & Erythromycin & 8 & 7 & 53.3 \\
Lincomycin & Clindamycin & 13 & 2 & 86.7 \\
Tetracyclines & Tigecycline & 1 & 14 & 6.7 \\
Penicillin & Tetracyclines & 0 & 15 & 0.0 \\
& Oxacillin & 0 & 15 & 0.0 \\
Fluoroquinolones & Benzylpenicillin & 0 & 15 & 0.0 \\
& Ciprofloxacin & 0 & 15 & 0.0 \\
& Levofloxacin & 0 & 15 & 0.0 \\
\hline
\end{tabular}

Table 5. Distribution of antibiotic sensitivity to staphylococcus epidermidis $(n=14)$

\begin{tabular}{llccc}
\hline Antibiotic Group & Antibiotics & Sensitive & Resistant & $\begin{array}{c}\text { Sensitive antibiotics } \\
\text { (\%) }\end{array}$ \\
& & 14 & 0 & 100.0 \\
Glycopeptides & Vancomicin & 14 & 0 & 100.0 \\
Furadantin & Nitrofurantoin & 14 & 0 & 100.0 \\
Antimicobacterial & Rifampicin & 14 & 0 & 100.0 \\
Oxazolidinone & Linezolide & 13 & 1 & 92.9 \\
Aminoglycosides & Gentamicin & 13 & 1 & 92.9 \\
Tetracyclines & Tigecycline & 7 & 7 & 50.0 \\
& Tetracyclines & 6 & 8 & 42.9 \\
Trimetorpim & Trimethoprim / Sulfamethoxazole & 5 & 9 & 35.7 \\
Fluoroquinolones & Moxifloxacin & 4 & 10 & 28.6 \\
& Ciprofloxacin & 4 & 10 & 28.6 \\
Macrolides & Levofloxacin & 3 & 11 & 21.4 \\
Lincomycin & Erythromycin & 1 & 4 & 20.0 \\
Penicillin & Clindamycin & 3 & 11 & 21.4 \\
& Oxacillin & 0 & 14 & 0.0 \\
\hline
\end{tabular}




\begin{tabular}{llccc} 
Antibiotic group & Antibiotics & Sensitive & Resistant & $\begin{array}{c}\text { Sensitive Antibiotics } \\
\text { (\%) }\end{array}$ \\
& & & 0 & 100.0 \\
\hline Aminoglycosides & Gentamicin & 11 & 0 & 100.0 \\
Fluoroquinolones & Ciprofloxacin & 11 & 0 & 100.0 \\
& Levofloxacin & 11 & 0 & 100.0 \\
& Moxifloxacin & 11 & 0 & 100.0 \\
Furadantin & Nitrofurantoin & 11 & 0 & 100.0 \\
Trimetorpim & Trimethoprim / Sulfamethoxazole & 11 & 100.0 \\
Antimicobacterial & Rifampicin & 11 & 0 & 100.0 \\
Oxazolidinone & Linezolide & 11 & 0 & 100.0 \\
Tetracyclines & Tigecycline & 11 & 0 & 54.5 \\
& Tetracyclines & 6 & 5 & 100.0 \\
Penicillin & Oxacillin & 11 & 0 & 9.1 \\
& Benzylpenicillin & 1 & 10 & 90.9 \\
Macrolides & Erythromycin & 10 & 1 & 90.9 \\
Lincomycin & Clindamycin & 10 & 1 & 90.9 \\
Glycopeptides & Vancomycin & 10 & 1 & \\
\hline
\end{tabular}

Table 7. Comparison of germ patterns between CSOM with cholesteatoma and CSOM without cholesteatoma

\begin{tabular}{|c|c|c|c|}
\hline \multirow{2}{*}{ Germ Pattern } & \multicolumn{2}{|c|}{$\mathrm{CSOM}$} & \multirow{2}{*}{ Total } \\
\hline & Cholesteatom & No Cholesteatom & \\
\hline Gram positive & $19(36.5)$ & $33(63.5)$ & $52(100)$ \\
\hline Gram negaitive & 45 (64.3) & 25 (35.7) & 70 (100) \\
\hline
\end{tabular}

${ }^{*}$ Chi-square pearson

Table 8. Multivariate analysis of factors affecting the incidence of cholesteatoma

\begin{tabular}{lcccc}
\hline Factor & $\begin{array}{c}\text { Coefficient } \\
\text { (B) }\end{array}$ & $\begin{array}{c}\boldsymbol{P} \\
\text { value }\end{array}$ & OR & CI 95\% \\
\hline Germ Group (Gram) & 1.140 & 0.003 & 3.126 & $1.482-6.597$ \\
\hline Constant & -1.692 & 0.007 & 0.184 & \\
\hline
\end{tabular}

\section{Discussion}

Based on the age category, the most research subjects were the age group 19-60 years, both in the CSOM group with cholesteatoma and the CSOM group without cholesteatoma, with an age range of 6 years to 70 years. Based on gender, there are more males than females with a ratio of 1.4: 1 . Based on the age group, the results of this study are slightly different from the 2019 study by Abarham which states that the proportion of CSOM is highest in the age group between 11-15 years and the least is in the age group over 40 years, while based on gender, the same results are found stating For 79 samples, the prevalence of men $(54.4 \%)$ was higher than that of women. In contrast to the descriptive epidemiological study by Kumari in 2019, from 60 samples of CSOM patients, 68.33\% were women with a male to female ratio of $1: 2$ and CSOM was more common in the 18-50 year age group. CSOM can occur in the age group of children because children often experience upper respiratory tract infections, immature immunity and the structure of the Eustachian tube which is shorter and more horizontal 
when compared to adults. This study used subject selection with total sampling, the dominance of male sex may be a coincidence finding, there are no known anatomical or genetic differences between men and women associated with ear infections, especially CSOM.2,6

The success of treating bacterial infections is the result of working between antibiotics and the immune system. Antibiotic resistance and the immune system are related to infection, the adaptation of bacteria to the immune system affects the spectrum of antibiotic resistance so that bacteria can become more resistant. Important determinants of the immune system include nutritional status and comorbid diseases. The nutritional status of 102 subjects who were the samples of this study showed that 66 subjects $(64.7 \%)$ had good nutritional status, 21 subjects with over nutritional status and 15 others with less nutritional status. In this study, nutritional status was determined from the medical records of patients both outpatient and inpatient who had anthropometric data in the form of measurements of body weight, body length or height. Some epidemiological data also show that body weight is related to the level of risk of infection. Both obesity and underweight increase the risk of infection whereas normal body weight has the lowest risk of infection. Subjects with obesity or over nutrition have a higher risk of infection because obesity contributes to an increased incidence of chronic inflammation of the fat tissue. In addition, there is accumulation of fat in the lymphoid organs which damages lymphoid tissue, thus interfering with the production and distribution of leukocytes or anti-inflammatory from lymphoid tissue $7-10$

Based on the presence or absence of comorbids, from a total of 102 subjects in this study, 22.5\% were found to be comorbid and $77.5 \%$ had no comorbids. Comorbid is one of the variables in the study because the immune system is also influenced by other underlying factors such as chronic or comorbid diseases (heart disease, kidney failure, chronic infections, autoimmune diseases, diabetes mellitus, malignancy) are factors that provide immunomodulatory effects so that they can contribute to risk of infection. Patients with comorbidities and multimorbidity have a greater risk of antibiotic resistance than patients without comorbidities because of their increased susceptibility to infection, more frequent use of antibiotics and more susceptibility to infection in strains of bacteria that are already resistant to antibiotics. Diabetes and chronic lung, kidney and vascular disease are all thought to increase a patient's susceptibility to bacterial infection or increase the risk of infection-related harm. However, the level of risk varies according to comorbidity, severity of disease and how the treatment is managed.11,12

Existing research shows Pseudomonas aeruginosa is the most common bacteria found in CSOM, similar to the results of this study where Pseudomonas aeruginosa was the most common microorganism found. The results of Andrade et al's study in Brazil on 83 patients (125 ears) of CSOM, which compared the results of culture. in CSOM with cholesteatoma and without cholesteatoma. The results showed that in CSOM without cholesteatoma, the most bacteria found were S. aureus, Pseudomonas sp. In CSOM with cholesteatoma, the most frequent findings are anaerobic bacteria and Corynibacterium sp. Frequency of S. epidermidis, Klebisiela sp. and Streptococcus sp. the same between these two diseases. Gram negative groups are more common in CSOM with cholestetaoma, this strengthens the theory that among the bacteria found, Pseudomonas aeruginosa is considered the main cause of the progressive destruction of the middle ear and mastoid structures due to the toxins and enzymes it produces. The main component of the Gram-negative bacterial cell wall, lipopolysaccharide, is also found in higher concentrations in patients with cholesteatoma and active bone destruction. In the bivariate analysis of the comparison of the pattern of germs between the two CSOM groups, the results of the analysis showed that Gram negative was found more in the CSOM group with cholesteatoma than without cholesteatoma, with a value of $p=0.002$. It can be concluded that there was a significant difference in the germ pattern between the two CSOM groups where Gram negative was more dominant in the CSOM group with cholesteatoma. This is thought to be due to the 
ability of Gram negative bacteria to form biofilms. ${ }^{13-16}$

\section{Conclusion}

Pseudomonas aeruginosa is the most common bacteria found to cause CSOM, the other bacteria are Staphylococcus haemolyticus, Staphylococcus epidermidis and Staphylococcus aureus. Pseudomonas aeruginosa is sensitive to the antibiotics amikacin (93.2\%), meropenem (84.15\%), gentamicin (79.5\%), cefepime $(77.3 \%)$, ciprofloxacin and ceftazidime $(72.7 \%)$. While resistant to the antibiotics tigecycline and cefazoline. There is a significant difference in the germ pattern between CSOM with cholesteatoma and CSOM without cholesteatoma. Gram negative bacteria are more common in CSOM with cholesteatoma, while Gram positive bacteria are more common in CSOM without cholesteatoma. Age, sex, nutritional status and comorbid diseases were not found to be factors that significantly influence the incidence of cholesteatoma in CSOM. OMSK sufferers with Gram negative bacterial infection had the opportunity to experience CSOM with cholesteatoma with a risk of 3.126 times.

\section{References}

1. Brennan-Jones CG, Head K, Chong LY, Burton MJ, Schilder AGM, Bhutta MF. Topical antibiotics for chronic suppurative otitis media. Cochrane database of systematic reviews. 2020; (1): 1-9.

2. Abraham ZS, Ntunaguzi D, Kahinga AA, Mapondella KB, Massawe ER, Nkuwi EJ, et al. Prevalence and etiological agents for chronic suppurative otitis media in a tertiary hospital in Tanzania. BMC Res Notes. 2019; 12:429.

3. Fukuda K. Antimicrobial resistance global report on surveillance: resistance to antibacterial drugs. Geneva: WHO; 2014. 109-116.

4. Sedyaningsih ER. Peraturan Menteri Kesehatan Republik Indonesia Nomor 2406: pedoman umum penggunaan antibiotik. Jakarta: Kemenkes RI; 2011. 1-5.

5. Moeloek NF. Peraturan Menteri Kesehatan Republik Indonesia Nomor 8: program pengendalian resistensi antimikroba di rumah sakit. Jakarta: Kemenkes RI; 2015.10-13.
6. Kumari GN, Manoj. A cross sectional study on quality of life measurements for patients with chronic suppurative otitis media in a tertiary care centre - a chronic ear survey. International Journal of Contemporary Medical Research. 2019;6(10): J1-J6.

7. Titchenal A, Hara S, Caacbay NA, Yang Y, Revilla MK, Langfelder G, et al. Human Nutrition: body mass index, body fat content and fat distribution. Carolyn, editor. Manoa: 2020. 133-39.

8. Gjini E, Brito $\mathrm{PH}$. Integrating antimicrobial therapy with host immunity to fight drug resistant infections: classical vs adaptive treatment. PLOS Comput Biol. 2016;12(4):1-34.

9. Ramiro RS, Costa H, Gordo I. Macrophage adaptation leads to parallel evolution of genetically diverse Escherichia coli small colony variants with increased fitness in vivo and antibiotic collateral sensitivity. 2016.

10. Harjatmo TP, Par'i HM, Wiyono S. Penilaian status gizi: metode penilaian status gizi. Jakarta: Kemenkes RI; 2017.44-63.

11. Dobner J, Kaser S. Body mass index and the risk of infection - from underweight to obesity. Clin Microbiol Infect. 2018;24: 24-8.

12. Rockenschaub P, Hayward A, Shallcross L. Antibiotic prescribing before and after the diagnosis of comorbidity: a cohort study using primary care electronic health records. Clinical Infectious Diseases. 2020;71(7):e50-7.

13. Chole RA, Nason R. Ballenger's otorhinolaryngology head and neck surgery: chronic otitis media and cholesteatoma. Wackym PA, editor. USA: PMPH; 2016.808-34 p.

14. Andrade JE, Pererira SH, Negro J, Neto RG, Carvalho MR, Filho NAC. Bacterial findings found in the chronic otitis media secretion: Comparative study between cholesteatoma (OMCC) and simple chronic otitis media (SCOM). Intl. Arch. Otorhinolaryngol. 2009;13(3): 287-92.

15. Riedel S, Morse SA, Mietzner T, Miller S. Medical microbiology: The staphylococci, Pseudomonas. 
28 th ed. Jawetz, editor. New York: McGraw-Hill

Lange; $2019 . \quad$ 205-212.

16. Sharma D, Misba L, Khan AU. Antibiotics versus biofilm: an emerging battleground in microbial communities. Antimicrobial resistance and infection control. 2019; 8:76. 